\title{
Multi-objective optimization strategy for distribution network considering V2G- enabled electric vehicles in building integrated energy system
}

\author{
Zhao Huang ${ }^{1,2^{*}}$, Baling Fang ${ }^{2}$ and Jin Deng ${ }^{1}$
}

\begin{abstract}
Based on the large-scale penetration of electric vehicles (EV) into the building cluster, a multi-objective optimal strategy considering the coordinated dispatch of EV is proposed, for improving the safe and economical operation problems of distribution network. The system power loss and node voltage excursion can be effectively reduced, by taking measures of time-of-use (TOU) price mechanism bonded with the reactive compensation of energy storage devices. Firstly, the coordinate charging/discharging load model for EV has been established, to obtain a narrowed gap between load peak and valley. Next, a multi-objective optimization model of the distribution grid is also defined, and the active power loss and node voltage fluctuation are chosen to be the objective function. For improving the efficiency of optimization process, an advanced genetic algorithm associated with elite preservation policy is used. Finally, reactive compensation capacity supplied by capacitor banks is dynamically determined according to the varying building loads. The proposed strategy is demonstrated on the IEEE 33-node test case, and the simulation results show that the power supply pressure can be obviously relieved by introducing the coordinated charging/discharging behavior of $\mathrm{EV}_{;}$in the meantime, via reasonable planning of the compensation capacitor, the remarkably lower active power loss and voltage excursion can be realized, ensuring the safe and economical operation of the distribution system.
\end{abstract}

Keywords: Distribution network, Electric vehicles, Multi-objective optimization, Coordinated dispatch, Advanced genetic algorithm

\section{Introduction}

With the continuous development of energy industry, the utilization of renewable energy as an alternative for fossil fuels has become a common sense of some countries [1]. Recently, the integrated energy system (IES) has been greatly supported by the Chinese government and turned into a research hotspot, since it benefits the integration of renewable energy, and the coordinated development of energy system. As the terminal node of IES, the building integrated energy system (BIES) provides customers with flexible cascades of different local energy resources, not only facilitating the environmentally

\footnotetext{
* Correspondence: skyhuang1985@hotmail.com

'Hunan college of information, Changsha, China

${ }^{2}$ College of electric and information engineering, Hunan university of technology, Zhuzhou, China
}

friendly, but also reducing the cost for consumers simultaneously $[2,3]$. The consumers in the building can be classified as two types: stationed institutions, and electric vehicles (EV) owners. EV is an important part for the future power system, for the more and more intense coupling with BIES. However, with the large-scale penetration of $\mathrm{EV}$, operation of the distribution network is enormously impacted by these random charging loads, since problems such as harmonic pollution, three-phase voltage imbalance, and aging of the transformers are induced. Especially, the enlarged load peak-valley gap puts forward higher requirements for the power system. The node voltage excursion as well as the active power loss is usually regarded as a crucial indicator reflecting the operation state of the distribution system. The peak load arisen from the aggregated charging behavior of EV 
owner probably leads to low voltage even collapse, concurrently increased system loss. Hence, guiding measures for coordinated regulation should be taken, to minimize the negative influences of EV while satisfying the travel demands of customers.

As part of a vehicle to grid (V2G) system [4], EV also can be regarded as controllable power resource, to realize the bidirectional power flow between the building and distribution system. Additionally, time-of-use (TOU) price mechanism is an effective method for the integrated demand side management, which creates an economic incentive for users to adjust their charging/ discharging time. For example, residential customers plug in their vehicles during off-peak hours. This helps to reduce the potential of distribution transformer overloads, as well as stabilize the load fluctuation by valley filling and peak shaving, thus balance the demand and supply.

A voltage regulation model is proposed after regulation of EV devices in [5], to reduce the voltage fluctuation of the power system, while the active loss has not been included in the optimization objective. Contrastively, the optimized model for distribution system reconfiguration is analyzed in [6], with the only optimization function of power loss. Researches about energy management and optimized operation of EV based on microgrid are also explored in many papers [7, 8]. Reference [9] similarly establishes a coordinated scheduling model of EV in response to TOU price. The charging/discharging plan is generated with fixed peak and valley periods, which maybe not suitable for the time-varying loads.

In this paper, the standard IEEE 33-node system is taken as an example, and measures of TOU price combined with the reactive power compensation devices are adopted. We select the tradeoff between power loss and node voltage excursion as the optimal objective. Under the designated simulation circumstance, the optimal compensation capacity is dynamically solved by an improved genetic algorithm. Through the peak shaving and valley filling effects on the building load curve of coordinated regulation of $\mathrm{EV}$, the positive guiding role of $\mathrm{TOU}$ price can be directly manifested. EV as controllable load inside BIES can participate in energy management effectively. Besides, by statistical results comparison of power loss and relative voltage excursion, the feasibility of reactive power compensation approach is also verified.

\section{Modeling of coordinated dispatch for EV}

In the presence of large-scale penetration of EV into BIES, the load pressure, operating cost, and reliance on distribution grid will be increased. Coordinated dispatch facilitates to boost the utilization of EV and improve the demand side flexibility. Due to the intrinsic advantages such as operational safety and energy density, the lithium ion batteries of $25 \mathrm{kWh}$ are selected as the power battery for EV [10-12], and the charging power maintains at $2.5 \mathrm{~kW}$ for a single vehicle. Concurrently, the safety threshold of the state of charge (SOC) is set to be $[10 \%, 90 \%]$, hence it can be inferred that the charging time could not be more than $8 \mathrm{~h}$. As the traffic infrastructure, supposing that power consumption for $\mathrm{EV}$ is $15 \mathrm{kWh}$ per $100 \mathrm{~km}$ [13], and the theoretical endurance mileage can be calculated as $133 \mathrm{~km}$.

\subsection{Structure of the BIES}

BIES is mostly configured as an energy cascade utilization system which enables free power flow among different energy types. The building cluster provides power to residents through external distribution power grid. As illustrated in Fig. 1, the demand side is comprised of three kinds of loads (cooling load, heating load, power load) and EV connections. EV devices are not considered as traditional loads since they can also act as a power supplier. For the V2G application, EV charging devices not only provide customers with charging services, but also allow the EV owners to sell excessive electricity to the power system.

\subsection{Aggregated charging of EV}

Aggregated charging of EV could be explained as charging behavior only according to customers' travel needs or living habits, without any guiding principle. As the EV travel follows a probability density distribution represented in Fig. 2:

$$
f_{d}(x)=\frac{1}{x \sigma_{d} \sqrt{2 \pi}} \exp \left[-\frac{\left(\ln x-\mu_{d}\right)^{2}}{2 \sigma_{d}^{2}}\right]
$$

where $\mu_{d}=3.20, \quad \sigma_{d}=0.88, \quad x$ denotes the daily mileage of EV (mostly lies between 32 and $97 \mathrm{~km}$ ), so a charged $25 \mathrm{kWh}$ battery can provide sufficient

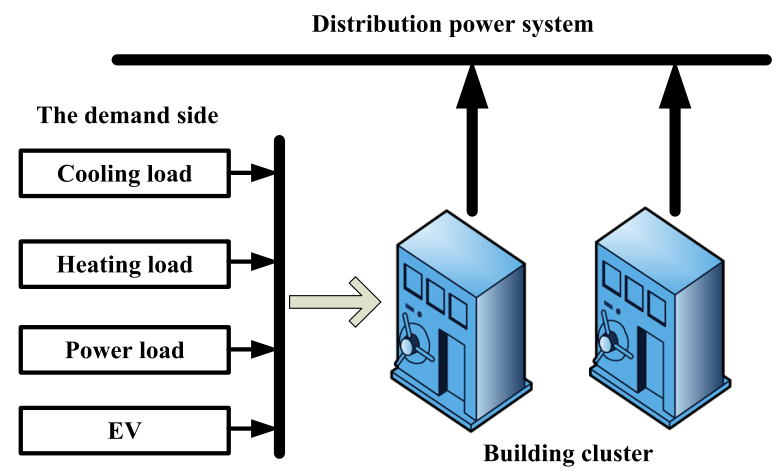

Fig. 1 Framework of BIES 


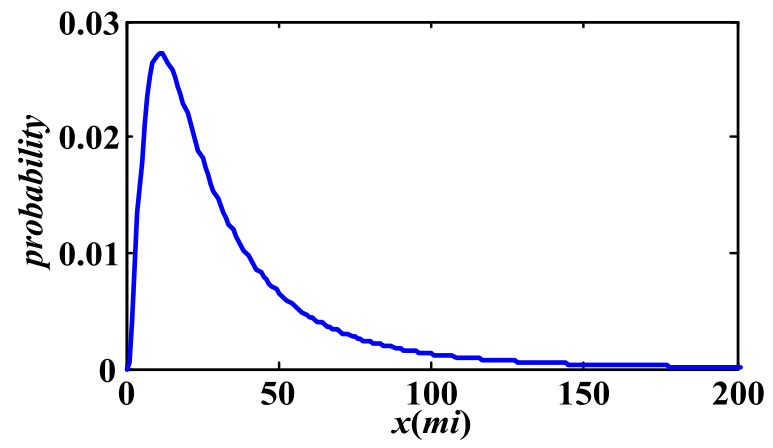

Fig. 2 Probability of driving distance during 1 day

energy to meet daily driving requirements. Monte Carlo random sampling can be used to predict the charging power demand for a single EV during a day. In this paper, $24 \mathrm{~h}$ in a day is divided into 96 periods, with sampling the state of EV per $15 \mathrm{~min}$. The charging expectation for a single vehicle is shown in Fig. 3.

\subsection{Coordinated dispatch of EV}

The three-stage TOU price is designated to establish a positive guidance for the charging/discharging process of the EV owners. Depending on the load change of the power grid, the corresponding electricity price level of each period is confirmed, to achieve the peak shaving and valley filling. In the modeling process of TOU price, minimizing the peak valley difference of the total loads in building cluster is chosen as the optimization objective, and the application background is specified as follows:

(1) the battery power meets the driving demand of the EV owners, and there is no other power consumption behavior beside the normal travel;

(2) user charge and discharge without exceeding the safe range of the SOC, and $80 \%$ of the total owners participate in the coordinated dispatch $(\lambda=0.8)$;

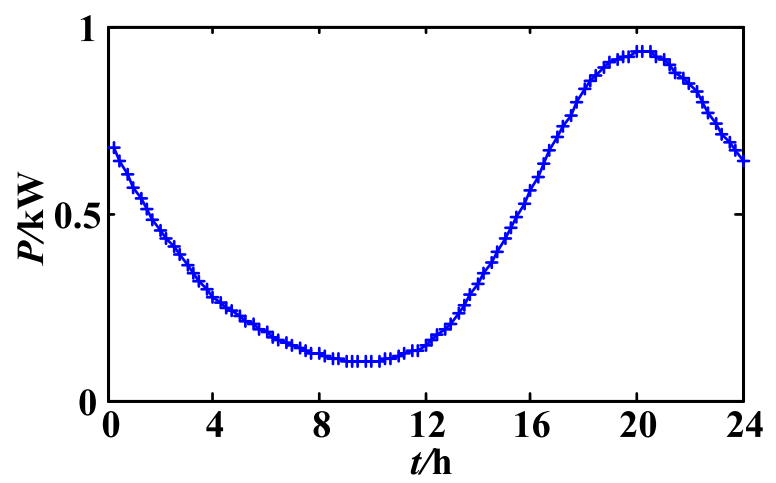

Fig. 3 Original charging requirement for a single EV
(3) $p_{v}, p_{\mathrm{p}}$ and $p_{\mathrm{f}}$ are defined as the electricity price of valley, peak and normal period respectively, then the price model $p(t)$ can be described as:

$$
p(t)= \begin{cases}p_{v} & t_{1} \leq t \leq t_{2} \\ p_{p} & t_{3} \leq t \leq t_{4} \\ p_{f} & \text { else }\end{cases}
$$

where $t_{1}-t_{4}$ represents the start and end moment of valley period, as well as peak period, respectively;

(4) before charging/discharging, the user can query the current battery status of the EV and independently select the charging/discharging time. The parameters $t_{s c}, t_{c}, t_{s d}, t_{d}$ are defined as follows:

$t_{s c}, t_{s d}$-the start moment for users to charge/discharge;

$t_{c}, t_{d}$-duration of charging/discharge process.

Users participating in the coordinated dispatch can select $t_{s c}$ or $t_{s d}$ according to Eqs. (3) and (4):

$$
\begin{aligned}
& t_{s c}= \begin{cases}t_{1}+\text { rand } \times\left(t_{2}-t_{c}\right) & 0 \leq t_{c} \leq t_{2}-t_{1} \\
t_{1} & \text { else }\end{cases} \\
& t_{s d}= \begin{cases}t_{3}+\text { randd } \times\left(t_{4}-t_{d}\right) & 0 \leq t_{d} \leq t_{4}-t_{3} \\
t_{3} & \text { else }\end{cases}
\end{aligned}
$$

where randc and randd are random numbers in $[0$, 1]. It can be known that for the TOU price model, parameters $t_{1}-t_{4}$ determine the peak and valley areas, and consequent daily load characteristics. Hence, they are key factors for optimal control of the distribution network.

\section{Modeling of distribution grid and var compensator}

The guiding ideology for the distribution network optimization can be concluded as follows: on the premise that the parameters such as branches and loads are known, and various constraints are satisfied, dynamically adjust the output of the pre-set capacitor bank to optimize the comprehensive indices of active power loss and voltage excursion. Finally, the economical and safe operation of the distribution power system can be achieved.

\subsection{Objective function}

The optimization objective function comprises two important issues: active power loss and voltage excursion of the distribution system. 


\subsubsection{Active power loss}

The first objective is the minimization of the total active power loss of the distribution network, which can be mathematically modeled as Eq. (5):

$$
\min f_{1}=\frac{\sum_{i=1}^{n} \sum_{j=1}^{n} P_{L i j}}{\sum_{i=1}^{n} \sum_{j=1}^{n} P_{L i j}^{\prime}}
$$

where $P_{L i j}^{\prime}, P_{L i j}$ respectively denote the active power loss of the branch $(i, j)$ before and after optimization.

\subsubsection{Voltage excursion}

Node voltage is an important indicator reflecting the security and service quality. To avoid all the voltages moving toward their maximum limits after optimization, the deviation of voltage from the rated value is chosen as an objective function, that is:

$$
\min f_{2}=\frac{\sum_{j=1}^{n}\left(U_{j}-U_{j \mathrm{~N}}\right)}{\sum_{j=1}^{n}\left(U_{j}^{\prime}-U_{j \mathrm{~N}}\right)}
$$

where $U_{j}, U_{j}, U_{j \mathrm{~N}}$ respectively represent the actual voltage of the node $j$ before and after optimization, and the rated voltage of the node $j$.

Combining the above two indicators, the objective function can be expressed as:

$$
\begin{aligned}
\min F= & \lambda_{1} \frac{\sum_{i=1}^{n} \sum_{j=1}^{n} P_{L i j}}{\sum_{i=1}^{n} \sum_{j=1}^{n} P_{L i j}^{\prime}}+\lambda_{2} \frac{\sum_{j=1}^{n}\left(U_{j}-U_{j \mathrm{~N}}\right)}{\sum_{j=1}^{n}\left(U_{j}^{\prime}-U_{j \mathrm{~N}}\right)} \\
& +\mu \sum_{j=1}^{n}\left(\frac{\Delta U_{j}}{U_{j \max }-U_{j \min }}\right)^{2}
\end{aligned}
$$

where $\lambda_{1}$ and $\lambda_{2}$ are the weight coefficients for the two optimization objectives $\left(\lambda_{1}=\lambda_{2}=0.5\right)$. The penalty function is used to deal with the node voltage out-oflimit problem, $U_{j \min }$ and $U_{j \max }$ are the minimum and maximum voltage values of the node $j$; while $\mu$ indicates the penalty factor $(\mu=1000)$, and $\Delta U_{j}$ is defined as (8):

$$
\Delta U_{j}=\left\{\begin{array}{lc}
U_{j}-U_{j \max } & U_{j}>U_{j \max } \\
0 & U_{j \min } \leq U_{j} \leq U_{j \max } \\
U_{j \min }-U_{j} & U_{j}<U_{j \text { min }}
\end{array}\right.
$$

\subsection{Constraints}

The basic constraints of distribution power system optimization mainly include equality constraints (power flow) and inequality constraints as follows:

A. SOC of lithium battery constraint:

$$
S O C_{\text {min }} \leq S O C \leq S O C_{\text {max }}
$$

where $S O C_{\min }$ and $S O C_{\max }$ are the safety threshold values of SOC, which are $10 \%$ and $90 \%$ respectively.

B. compensation capacity constraint:

$$
0 \leq C_{j}=k_{j} C_{j N} \leq C_{j \max }
$$

where $C_{j}$ and $C_{j \max }$ illustrate the output capacity of the capacitor bank installed on the node $j$, and its maximum capacity respectively; $C_{j \mathrm{~N}}$ is the capacity of a single capacitor included in the capacitor bank; $k_{j}$ is the number of single capacitors on the node $j$.

\subsection{Algorithm}

The purpose of multi-objective optimization is to determine a set of Pareto solutions, which compromise each optimization objective. Herein, a novel genetic algorithm based on elite preservation policy is introduced to solve the optimization mathematical model established in 3.1 and 3.2. Principally, the crossover probability $P_{c}$ and mutation probability $P_{m}$ are improved as follows [14]:

$$
\begin{aligned}
& P_{c}= \begin{cases}\frac{k_{1}\left(F_{\max }-F^{\prime}\right)}{F_{\max }-F_{\mathrm{avg}}} & F^{\prime} \geq F_{\mathrm{avg}} \\
k_{2} & F^{\prime}<F_{\mathrm{avg}}\end{cases} \\
& P_{m}= \begin{cases}\frac{k_{3}\left(F_{\max }-F^{\prime \prime}\right)}{F_{\max }-F_{\mathrm{avg}}} & F^{\prime \prime} \geq F_{\mathrm{avg}} \\
k_{4} & F^{\prime \prime}<F_{\mathrm{avg}}\end{cases}
\end{aligned}
$$

where $k_{1}-k_{4}$ are constant, which are set to be $0.5 、 0.9 、 0.02$ and 0.05 ; while $F_{\text {avg }} 、 F_{\max }, F^{\prime}$ and $F^{\prime \prime}$ respectively demonstrate the average and maximum fitness value of individuals, the better fitness of the two crossover individuals, and the fitness of the mutation individuals. By improving $P_{c}$ and $P_{m}$, it helps to duplicate the good individuals and eliminate the bad solutions. In order to improve the calculation efficiency, the elite preservation policy is taken into consideration. After the power flow calculation of the initial population, $20 \%$ of the individuals with the best compromise effects of network loss and voltage fluctuation are reproduced directly 
to the next generation, while rest individuals are randomly selected by the roulette wheel method and produced according to the crossover and mutation operations. The flowchart of the algorithm is shown in Fig. 4.

\section{Results and discussion}

The standard IEEE 33-node power distribution system shown in Fig. 5 is tested for verification, and its load information is detailed in reference [15]. The basic parameters of the network for the perunit system are 10 MVA and $12.66 \mathrm{kV}$. It is assumed that the building loads are evenly distributed on these four nodes: $3,10,18,32$. Considering the time-varying characteristics of the daily loads, a series of building load data including cooling load, heating load and power load, whereas without EV integration, for a certain day are collected in Fig. 6 (original building loads). Distributed generator DG1 and DG2 are respectively installed on the node 2 and 5, with the output of $1+\mathrm{j} 0.484$ MVA. The capacitor banks for compensation are installed on the node 17 and 32, with the maximum capacity of 1 Mvar. EV scale inside the buildings connected to the distribution grid is 500 , with the charge/ discharge power of $2.5 \mathrm{~kW}$. Furthermore, the convergence accuracy of power flow calculation is $10^{-4}$.

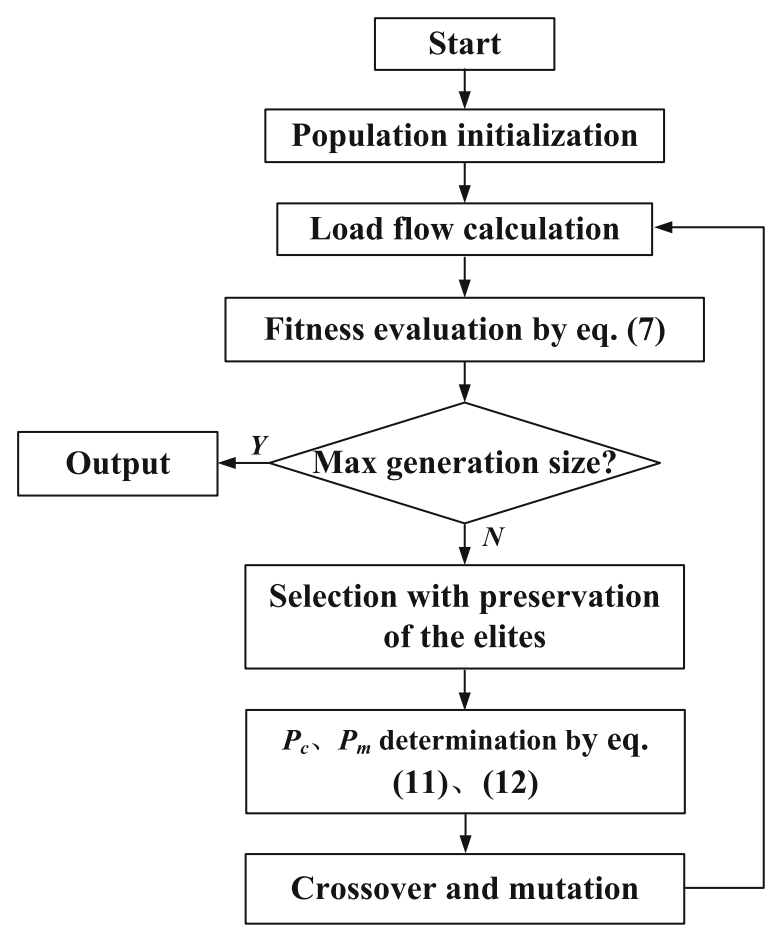

Fig. 4 Flowchart of the developed genetic algorithm

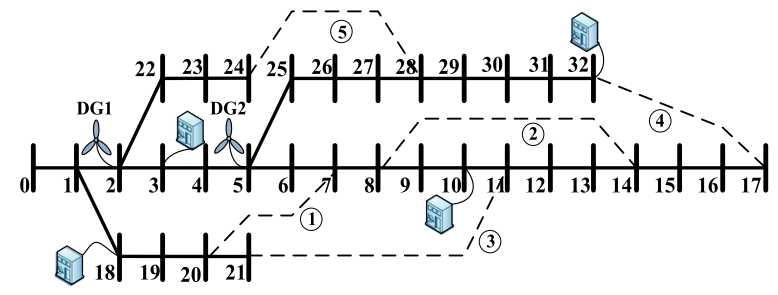

Fig. 5 IEEE 33-node system

Based on the building load curve in Fig. 6, the optimized result under the coordinated dispatch of EV is obtained, as depicted in Fig. 7. For comparison, the aggregated EV charging result is also exhibited. It can be observed that the load area between 17: 00-20:00 is intensified by the aggregated EV charging behavior, which puts forward higher requirements for the power supply. The attempt of coordinated charging/discharging regulation could realize the function of load peak shaving and valley filling, circumventing above problem. By introducing the TOU price, which makes full use of the schedulability of V2G, the load curve can be smoothed, while guaranteeing the charging demand of EV owners.

Concretely, the distribution system is most prone to black out at about 19:00 for the original building load curve, with the peak load of $3715 \mathrm{~kW}$. However, this would be further increased to 4167 $\mathrm{kW}$ under the access of aggregated EV charging. After coordinated regulation, the peak load can be optimized to $3608 \mathrm{~kW}$ (decreased by $13.41 \%$ ). Accordingly, voltages for all of the nodes are also calculated, as demonstrated in Fig. 8. Supposing that a voltage dip lower than 0.95 p.u. or a voltage higher than 1.05 p.u. is deemed as voltage out-oflimit, then it is discovered that under the EV aggregated charging, the system voltage drops seriously. Only 11 node voltages are better than the

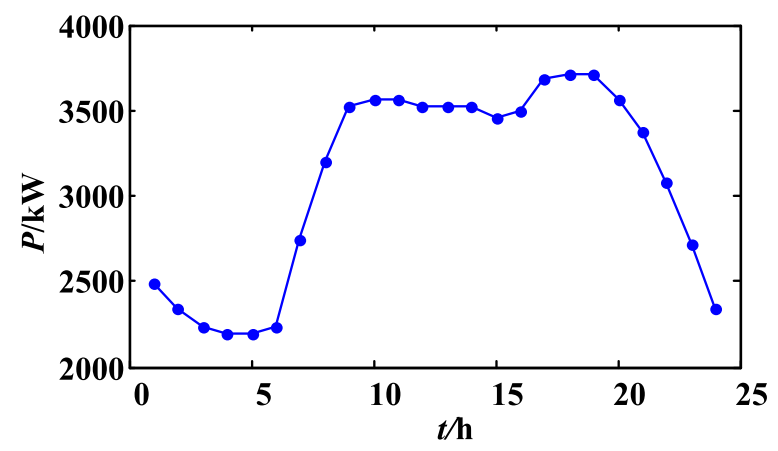

Fig. 6 Daily original building load curve 


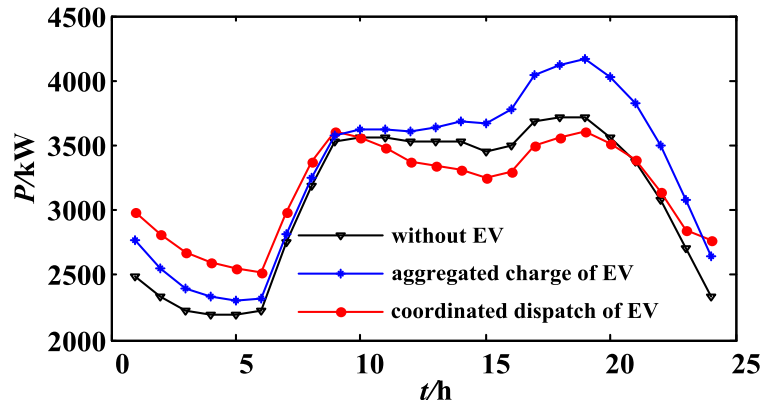

Fig. 7 Load curves under two regulation modes of EV

lower bound, and the worst one of 0.834 p.u. occurs on the node 32 . In contrast, the system voltage is overall heightened on coordinated regulation mode, and the voltage of the node 32 is raised up to 0.846 p.u., close to its value when the distribution system operates with original building loads (0.844 p.u.).

Moreover, through the power flow analysis, Table 1 illustrates the statistical results of the two indices for the system under different EV regulation modes. It's obvious that the integration of building loads increases the power supply burden of distribution system, since the problems such as power consumption and voltage quality are more serious. By the introduction of TOU mechanism, the coordinated management of flexible loads inside the buildings not only boosts the utilization of EV, but conducive to the economical and safe operation of the grid, consistent with the concept of energy conservation and emission reduction of modern power system.

DG1 and DG2 separately connected to the node 2 and 5 can further support the system voltage. After the DG input, 12 nodes exceed the voltage lower limit, the smallest value is still on the node 32,
Table 1 Indices under different EV regulation modes

\begin{tabular}{lll}
\hline Regulation mode & $\begin{array}{l}\text { Relative voltage excursion } \\
\text { (p.u.) }\end{array}$ & $\begin{array}{l}\text { Power loss } \\
(\mathrm{kW})\end{array}$ \\
\hline Without building loads & 1.701 & 202.6 \\
$\begin{array}{l}\text { With original building } \\
\text { loads }\end{array}$ & 3.019 & 666.9 \\
$\begin{array}{l}\text { Aggregated charging of } \\
\text { EV }\end{array}$ & 3.199 & 755.2 \\
$\begin{array}{l}\text { Coordinated dispatch of } \\
\text { EV }\end{array}$ & 2.978 & 647.2 \\
\hline
\end{tabular}

which is raised to 0.874 p.u. The relative voltage excursion of each node totally adds up to 2.327 p.u., decreased by $22.92 \%$ compared to 3.019 p.u. of the original building loads. Finally, by employing the improved genetic algorithm analyzed in 3.3, the optimal compensation capacities of the capacitor banks on the node 17 and 32 are solved as 0.95 and 0.81 Mvar respectively. The risk of voltage outof-limit is effectively reduced after reactive compensation, which can be testified by the node voltage of 32 (0.914 p.u.). Based on the coordinated dispatch of EV devices, Fig. 9 declares the node voltages of the distribution system before and after compensation, emphasizing an excellent effect of voltage fluctuation suppression. After reactive compensation by DG and capacitor banks, the total voltage excursion is optimized to 1.591 p.u. (decreased by $47.3 \%$ compared to that of the original building loads).

In addition, as shown in Table 2, when DG and capacitor are not installed, the active power loss of the system is $647.2 \mathrm{~kW}$; which is reduced to 411.9 $\mathrm{kW}$ when DG is put into operation but capacitor compensation is not available. After both of the DG and capacitor banks are put into use, the system loss is further decreased to $397.3 \mathrm{~kW}$. Compared with power consumption of $666.9 \mathrm{~kW}$ for the original building loads, the reduction achieves 2.95\%,

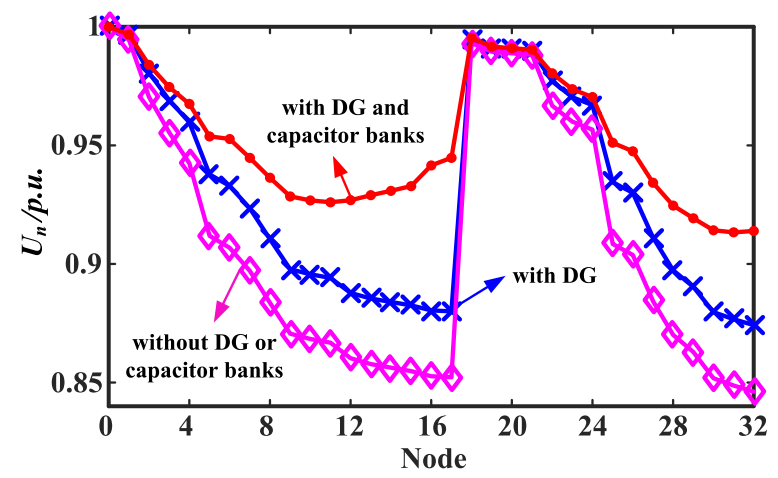

Fig. 9 Comparison results of node voltages before and after reactive compensation

Fig. 8 Voltage curves under two operation modes of EV 
Table 2 Indices before and after compensation

\begin{tabular}{lll}
\hline Compensation & $\begin{array}{l}\text { Relative voltage excursion } \\
\text { (p.u.) }\end{array}$ & $\begin{array}{l}\text { Power loss } \\
(\mathrm{kW})\end{array}$ \\
\hline $\begin{array}{l}\text { Without DG or capacitor } \\
\text { banks }\end{array}$ & 2.978 & 647.2 \\
$\begin{array}{l}\text { With DG } \\
\text { With DG and capacitor }\end{array}$ & 2.327 & 411.9 \\
banks & 1.591 & 397.3 \\
\hline
\end{tabular}

$38.2 \%$ and $40.4 \%$ respectively. Therefore, the reasonable dispatch of EV weakens the burden of the distribution network for power supply, concurrently the multi-objective optimization approach also ensures the significant improvements of system power loss and node voltage excursion, proving that the proposed strategy in this paper could obtain outstanding performance.

It's worth to note that the response degree of the EV users $\lambda$ also has an impact on the optimal effects for the distribution network. For the demand side, participating in regulation favors the economic interest of customers. For the network, a higher $\lambda$ definitely corresponds to a weaker difference between load peak and valley. This is conducive to improving both the initiative of users and security of the distribution system.

\section{Conclusions}

In dependence on the basic principle of optimal control for distribution network, considering the popularization of EV devices in BIES, an optimization strategy is put forward in this paper. The strategy combines the pricing mechanism in the buildings and the dynamic compensation of the energy storage device, which is designed to balance the optimization between system power loss and node voltage fluctuation. By simulation analysis and comparison on the MATLAB, the overall ability of the optimization strategy is illustrated. Firstly, BIES provides access for EV, enhancing more space for schedulable resource capacity. Next, introduction of EV coordinated dispatch by the TOU price mechanism promotes their initiative and utilization, and alleviates the power supply demand of the distribution network by load peak shaving and valley filling. Finally, under the condition of basic constraints, the optimal solution of compensation plan by capacitor banks can be determined through an improved genetic algorithm. After scheduled charging/discharging of EV and reactive compensation, the active power loss and risk of node voltage out-of-limit are improved simultaneously, promoting the economical and safe operation of the distribution network. This optimal strategy can be extended to other cases, and has great significance for the optimal management of power grid.

\section{Abbreviations}

BIES: Building integrated energy system; EV: Electric vehicles; IES: Integrated energy system; SOC: State of charge; TOU: Time-of-use; V2G: Vehicle to grid

\section{Authors' contributions \\ BF performed the literature review and collected the information pertaining to distribution power system optimization. JD provided critical comments and technical guidance for the algorithm. ZH performed the simulation, analyzed the results, and wrote the paper. All authors read and approved the final version of the manuscript.}

\section{Author information}

Zhao Huang (1985-), female, PHD and Lecturer, Major in the energy storage devices in power system, and distribution grid optimal operation control. Baling Fang (1980-), male, PHD and Lecturer, Major in the power network planning modeling, and power grid data mining research.

Jin Deng (1991-), female, MS and Lecturer, Major in the planning theory application in distribution network.

\section{Funding}

This work was supported by Natural Science Foundation of Hunan Province (2017JJ5044).

\section{Availability of data and materials \\ Not applicable.}

\section{Consent for publication}

Not applicable.

\section{Competing interests}

The authors declare that they have no competing interests.

Received: 31 October 2019 Accepted: 15 January 2020

Published online: 31 January 2020

\section{References}

1. Hu, W., Su, C., Chen, Z., et al. (2013). Optimal operation of plug-in electric vehicles in power systems with high wind power penetrations. IEEE Transactions on Sustainable Energy, 4(3), 577-585.

2. Qin, C., Yan, Q., \& He, G. (2019). Integrated energy systems planning with electricity, heat and gas using particle swarm optimization. Energy, 188, 116044.

3. Alshahrani, S., Khalid, M., \& Almuhaini, M. (2019). Electric vehicles beyond energy storage and modern power networks: challenges and applications. IEEE Access, 7, 99031-99064.

4. Wang, Z., \& Wang, S. (2013). Grid power peak shaving and valley filling using vehicle-to-grid systems. IEEE Transactions on Power Delivery, 28(3), 1822-1829.

5. Guo, Y., Xinhui, Z., Ke, P., et al. (2019). Voltage regulation strategy of distribution network considering the joint action of electric vehicle and photovoltaic power supply. Electrical Measurement \& Instrumentation, 56(7), 75-83.

6. Guang, C., Pan, D., Hao, Z., et al. (2013). Distribution system reconfiguration considering distributed generators and plug-in electric vehicles. Power System Technology, 37(1), 82-88.

7. Tan, L., Caihong, Z., Ziqi, C., et al. (2015). Economical dispatch for microgrids of electric vehicles and distributed power. Power System and Clean Energy, 31(4), 100-105.

8. Zhang, M., \& Chen, J. (2014). The energy management and optimized operation of electric vehicles based on microgrid. IEEE Transactions on Power Delivery, 29(3), 1427-1435.

9. Dajun, W., Chenghui, Z., Bo, S., et al. (2014). A time-of-use price based multiobjective optimal dispatching for charging and discharging of electric vehicles. Power System Technology, 38(11), 2972-2977.

10. Ziemann, S., Müller, D. B., Schebek, L., et al. (2018). Modeling the potential impact of lithium recycling from EV batteries on lithium demand: A dynamic MFA approach. Resources, Conservation and Recycling, 133, 76-85.

11. Zhang, P., Qian, K., Zhou, C., et al. (2012). A methodology for optimization of power systems demand due to electric vehicle charging load. IEEE Transactions on Power Systems, 27(3), 1628-1636. 
12. Nykvist, B., \& Nilsson, M. (2015). Rapidly falling costs of battery packs for electric vehicles. Nature Climate Change, 5(4), 329.

13. Liang, Z., Zheng, Y., Donghan, F., et al. (2014). Two-stage optimization model based coordinated charging for EV charging station. Power System Technology, 38(4), 967-973.

14. Ruiz-Rodriguez, F. J., Hernández, J. C., \& Jurado, F. (2018). Voltage behaviour in radial distribution systems under the uncertainties of photovoltaic systems and electric vehicle charging loads. International Transactions on Electrical Energy Systems, 28(2), e2490.

15. Tsai, C. C., Huang, H. C., \& Chan, C. K. (2011). Parallel elite genetic algorithm and its application to global path planning for autonomous robot navigation. IEEE Transactions on Industrial Electronics, 58(10), 4813-4821.

\section{Submit your manuscript to a SpringerOpen ${ }^{\circ}$ journal and benefit from:}

- Convenient online submission

- Rigorous peer review

- Open access: articles freely available online

High visibility within the field

- Retaining the copyright to your article

Submit your next manuscript at $\boldsymbol{\nabla}$ springeropen.com 